\title{
Assessment of bone status using the contact ultrasound bone analyser
}

\author{
M Z Mughal, K Ward, N Qayyum, C M Langton
}

\begin{abstract}
Broadband ultrasound attenuation (BUA) was measured at the calcaneum in 367 healthy white schoolchildren (193 girls and 174 boys) aged 6-15 years. The mean calcaneal (BUA) increased with age and was significantly related to age, height, and weight. Measurement of calcaneal (BUA) may be helpful in the radiation free assessment of childhood disorders associated with increased fracture risk.

(Arch Dis Child 1997;76:535-536)
\end{abstract}

Keywords: broadband ultrasound attenuation; contact ultrasound bone analyser; bone mineral density; dual energy $x$ ray absorptiometry

Quantitative ultrasound technology is increasingly being used for the assessment of postmenopausal osteoporosis. ${ }^{1}$ The principle behind such measurements is that the speed at which ultrasound waves pass through bone, or the loss of their energy by attenuation mechanisms such as scattering and absorption, are proportional to both the physical density and structural properties of bone. We have shown that the broadband ultrasound attenuation at the calcaneum (calcaneal BUA) correlates well $(r=0.74, \mathrm{p}<0.001)$ with the total body bone mineral density (BMD) measured by dual energy $x$ ray absorptiometry (DXA) in healthy children $^{2}$ and moderately well $(r=0.60, \mathrm{p}=$ 0.01 ) with the spinal BMD measured by quantitative computed tomography in children with cerebral palsy. ${ }^{3}$ In postmenopausal women, Bauer et al reported an association between low calcaneal BUA and vertebral fractures, which persisted even after adjustment for $\mathrm{BMD}{ }^{1}$ These workers concluded that calcaneal BUA provides information about fracture risk, which is, in part, independent of the BMD.

The objectives of this cross sectional study were to establish the reference ranges for calcaneal BUA in 6-15 year old white children and to examine the relations between calcaneal BUA and age, height, and weight.

\section{Patients and methods}

We studied 367 healthy schoolchildren (193 girls and 174 boys) aged 6-15 years. Children participating in this study were recruited from inner city schools in Manchester and Sheffield. Ethical approval for the study was obtained from the Manchester and Sheffield medical research ethics committees. Written informed consent was obtained from the parents and verbal consent from the children. Any child who had a chronic medical disorder or who was taking drugs known to affect the BMD was excluded.

Height was measured to the nearest millimetre using a wall mounted height measuring tape (Raven Equipment, Great Dunmow, Essex). Each child was weighed to the nearest $0.5 \mathrm{~kg}$ while wearing their school uniform without shoes or socks. Pubertal assessment was not allowed by the medical research ethics committees.

The BUA $(\mathrm{dB} / \mathrm{MHz})$, which reflects the frequency dependence of ultrasound attenuation in the frequency range $200-600 \mathrm{kHz}$, was measured in triplicate at the left calcaneum of the subjects using the prototype paediatric contact ultrasound bone analyser (CUBA, McCue Ultrasonics Ltd, Compton, Winchester) as described previously. ${ }^{2}$ The central 'waist' of the calcaneum is chosen as the site for the measurement of BUA (calcaneal BUA) as it is readily accessible and consists of $>90 \%$ metabolically active trabecular bone. The in vitro and in vivo (in 6-18 year olds) coefficients of variation for the paediatric CUBA are 1.7 and $5 \%$, respectively.

The SPSS (version 6) package for Microsoft Windows was used for statistical analysis. Normative values for BUA were produced for boys and girls for each one year age range between the ages of 6 and 15 years. Pearson correlation
Paediatrics, St Mary's Hospital, Hathersage

Road, Manchester

M13 0JH

M Z Mughal

K Ward

N Qayyum

Departments of Medical Physics, University of Hull and

Royal Hull Hospitals

Trust, Hull

C M Langton

Correspondence to: Dr Mughal.

Accepted 11 February 1997
Table 1 Height, weight, and BUA in boys and girls expressed as mean (SD)

\begin{tabular}{|c|c|c|c|c|c|c|c|c|}
\hline \multirow[b]{2}{*}{ Age (years) } & \multicolumn{4}{|c|}{ Boys } & \multicolumn{4}{|c|}{ Girls } \\
\hline & No & Height $(\mathrm{cm})$ & Weight (kg) & $\begin{array}{l}B U A(d B / \\
M H z)\end{array}$ & No & Height (cm) & Weight (kg) & $\begin{array}{l}B U A(d B / \\
M H z)\end{array}$ \\
\hline $6-7$ & 18 & $120.4(4)$ & $24.4(3)$ & $40.9(10)$ & 25 & $121.7(5)$ & $24.2(4)$ & $35.5(11)$ \\
\hline $7-8$ & 13 & $127.2(6)$ & 27.5 (3) & 42.9 (11) & 20 & $125.7(5)$ & 27.0 (4) & 39.2 (11) \\
\hline $8-9$ & 20 & $131.5(7)$ & $31.0(6)$ & $45.2(13)$ & 33 & $132.2(5)$ & $30.7(5)$ & $45.8(11)$ \\
\hline $9-10$ & 27 & $136.2(6)$ & $33.3(6)$ & 59.7 (18) & 30 & $137.7(5)$ & $31.2(5)$ & $51.6(13)$ \\
\hline $10-11$ & 22 & $140.9(6)$ & $35.4(6)$ & $60.4(16)^{\star}$ & 26 & $142.0(8)$ & $37.5(10)$ & $47.8(12)$ \\
\hline $11-12$ & 19 & $149.0(9)$ & $42.4(10)$ & $72.1(18)$ & 12 & $149.4(8)$ & $43.4(9)$ & $65.2(18)$ \\
\hline $12-13$ & 19 & $157.6(8)$ & $50.2(11)$ & $80.3(18)^{\star}$ & 24 & $156.6(8)$ & 49.9 (11) & 65.9 (18) \\
\hline $13-14$ & 25 & $161.1(10)$ & $53.9(12)$ & $76.4(21)$ & 17 & $160.2(5)$ & $53.6(11)$ & $70.5(15)$ \\
\hline $14-15$ & 10 & $167.9(6)$ & $62.4(12)$ & $87.9(16)$ & 6 & $158.4(7)$ & $62.6(8)$ & $74.6(16)$ \\
\hline
\end{tabular}




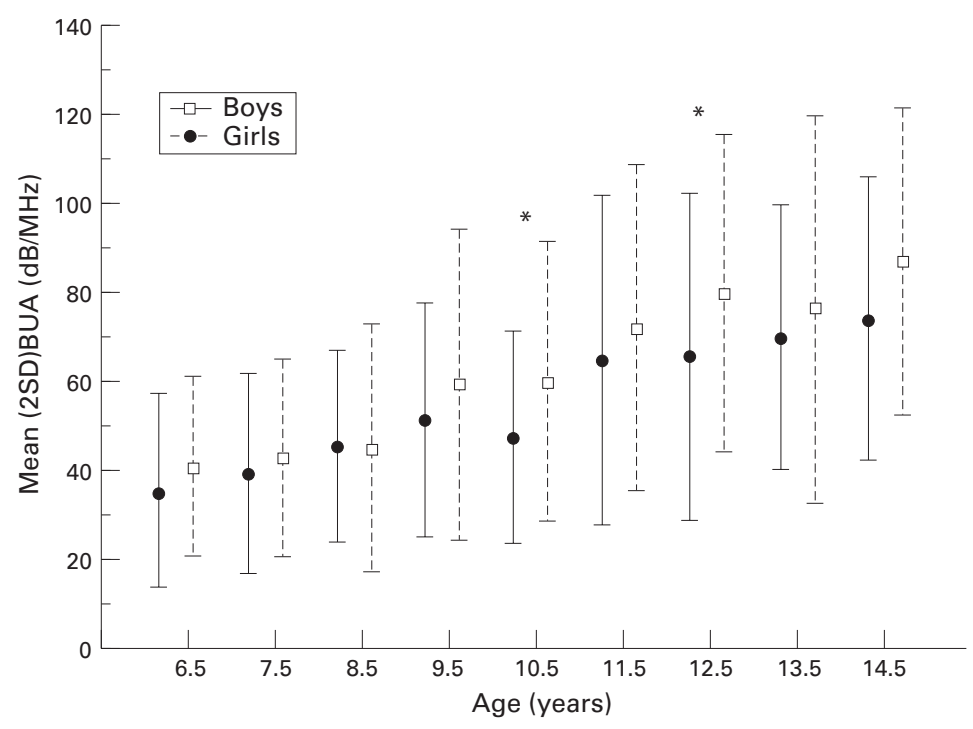

Figure 1 Relation between age (midpoint of age ranges in table 1) and mean (2SD) calcaneal BUA values; ${ }^{\star} p<0.01$ (unpaired Student's $t$ test).

coeffieients between BUA, age, height, and weight were calculated using linear regression analyses.

\section{Results}

The details of the mean (SD) height, weight, and calcaneal BUA are shown in table 1. As shown in fig 1 , the boys generally had higher mean calcaneal BUA values than the girls, but this was only significantly different $(p<0.01)$ in the age ranges $10-11$ and $12-13$. For the combined group significant $(\mathrm{p}<0.001)$ positive correlations were found between the mean calcaneal BUA $(\mathrm{dB} / \mathrm{MHz})$ and age $(r=0.67, \mathrm{BUA}=$ $(5.6 \times$ age in years -2.4$))$, height $(r=0.71$, BUA $=(0.96 \times$ height in $\mathrm{cm}-78))$, and weight $(r=0.73, \mathrm{BUA}=(1.12 \times$ weight in $\mathrm{kg}+14.1))$.

\section{Discussion}

The main aim of this study was to establish reference ranges for calcaneal BUA measured by the prototype paediatric CUBA in 6-15 year old children. Only white children were included in this study because of the effects of ethnicity on BMD. ${ }^{4}$ The results show that the mean calcaneal BUA values increased continu- ously between 6 and 15 years of age and were significantly related to age, height, and weight. These findings are in agreement with other studies in which BMD in children was measured by DXA. ${ }^{5}$ Reference values for calcaneal BUA in children are, however, dependent on the commercial ultrasound bone densitometer used and mathematical adjustments made to the true calcaneal BUA. Our values for the calcaneal BUA are lower than those reported by Jaworski et al, who used the Achilles ultrasound densitometer (Lunar Corporation, Madison, WI, USA) to measure the calcaneal BUA in 6-13 year old children. ${ }^{6}$

Jaworski et al found that the calcaneal BUA discriminated healthy children from those known to have osteoporosis (mean total body BMD SD score of $-3.3(0.9)) .^{6}$ Although the paediatric CUBA cannot be used to diagnose osteoporosis, which by convention is defined in terms of BMD, the availability of reference data for calcaneal BUA should allow the evaluation of the paediatric CUBA as a screening tool to identify those children who have increased fracture risk - for example, those treated with systemic steroids. The paediatric CUBA is a relatively low cost, portable, and radiation free system with a scan time of less than three minutes. It is therefore eminently suitable for use in paediatric outpatient clinics, schools, and in field studies.

We thank all the children who took part in the study and the staff at the participating schools. We also thank Dr I M Doughty and Dr S W D'Souza for their helpful comments.

1 Bauer DC, Gluer CC, Genant HK, Stone K. Quantitative ultrasound and vertebral fracture in postmenopausal women. F Bone Miner Res 1995;10:353-8.

2 Mughal MZ, Langton CM, Utretch G, Morrison J, Specker $\mathrm{BL}$. Comparison between broadband ultrasound attenuation of the calcaneum and total body bone mineral density in children. Acta Paediatr 1996;85;6635.

3 Wilmshurst S, Ward K, Adams JE, Langton CM, Mughal MZ. Mobility status and bone density in cerebral palsy. Arch Dis Child 1996;75:164-5.

4 Gilsanz V, Roe TF, Mora S, Costin G, Goodman WG. Changes in vertebral bone density in black girls and white girls during childhood and puberty. $N$ Engl $f \mathrm{Med}$ 1991;325:1597-600.

5 Glastre C, Braillon P, David L, Cochat P, Meunier PJ, Delmas PD. Measurement of bone mineral content of the lumbar spine by dual energy x-ray absorptiometry in normal children: correlations with growth parameters. $\mathcal{F}$ Clin Endochildren: correlations with grow

6 Jaworski M, Lebiedowski M, Lorenc RS, Trempe J. ultrasound bone measurement in pediatric subjects. Calcif Tissue Int 1995;56:368-71. 